\title{
Dolor sacroilíaco: Epidemiología, fisiopatología, diagnóstico y tratamiento
}

\author{
GERARDO CORREA-ILLANES ${ }^{1}$.
}

\section{ABSTRACT \\ Sacroiliac pain: epidemiology, physiopathology, diagnosis and treatment}

Sacroiliac pain is an frecuent underdiagnosed source of low back pain, affecting $15 \%$ to $30 \%$ of individuals with chronic, non-radicular pain. The sacroiliac joint (SIJ) is subject to continuous stress during standing position and gait, being stabilized by strong ligament, capsular and myofascial structures with rich innervation. Due to its heterogeneous nature, SIJ pain is difficult to diagnose, and it should be suspected in all patients with non-radicular unilateral and non-central low back pain syndrome. Physical examination should rule out hip and lumbar spine pathology. SIJ provocation maneuvers are used for diagnosis, with the combination of 3 or more positive maneuvers resulting in a sensitivity of $85 \%$ and a specificity of $79 \%$. Diagnostic injections of local anesthetics, both intra-articular and in the surrounding ligaments have been used. Treatment of SIJ pain is multimodal and individualized for each patient. Conservative treatment, based on physical therapy and nonsteroidal anti-inflammatory drugs (NSAIDs) is the first line therapy. Both intra- and extra-articular steroid infiltrations can provide relief in a group of patients with active inflammation. Radiofrequency denervation of lateral dorsal branches has proven to be a successful treatment in SIJ pain patients, achieving 6 to 12 months of pain relief. In patients with refractory pain, SIJ fusion is an option, with minimally invasive trans-sacroiliac fixation being the preferred technique.

Key words: Low back pain, sacroiliac joint dysfunction, sacroiliac joint pain, sacroiliac joint denervation, intra-articular injection, sacroiliac joint injection.

\section{RESUMEN}

El dolor sacroilíaco es una causa generalmente subdiagnosticada de dolor lumbar, que afecta del $15 \%$ a $30 \%$ de los pacientes con dolor lumbar bajo crónico no radicular. La articulación sacroilíaca (ASI) recibe continuo stress durante la bipedestación y marcha, siendo estabilizada por estructuras ligamentarias, capsulares y miofasciales fuertes, que reciben una abundante inervación. Destaca la dificultad en el diagnóstico del dolor sacroilíaco; debi-
'Unidad de Dolor, Instituto de Neurorrehabilitación (INER).

Hospital Clínico Mutual de Seguridad CChC.

Correspondencia: gerardo.correa01@gmail.com

Recibido: 19-11-2019 Aprobado: 30-12-2019 
do a su naturaleza heterogénea. Éste se debe sospechar en todo paciente con síndrome de dolor lumbar no radicular, unilateral y no central. El examen físico debería descartar patología de cadera y columna lumbar. La realización de maniobras de provocación del dolor sacroilíaco aporta en el diagnóstico, teniendo la combinación de 3 o más maniobras positivas una sensibilidad de $85 \%$ y especificidad de $79 \%$. Se ha recurrido a inyecciones diagnósticas con anestésicos locales, tanto intraarticulares como de ligamentos circundantes. El tratamiento del dolor sacroilíaco es multimodal e individualizado para cada paciente. El tratamiento conservador-basado en terapia física y antiinflamatorios no esteroidales- es la terapia de primera línea. Las infiltraciones esteroidales tanto intra como extraarticulares pueden proveer alivio en un grupo de pacientes con inflamación activa. La denervación de los ramos dorsales laterales con radiofrecuencia ha mostrado ser un tratamiento exitoso en pacientes con dolor sacroilíaco, logrando 6 meses a 1 año de alivio del dolor. En pacientes con dolor refractario, la fusión de la articulación sacroilíaca es una opción, prefiriéndose la técnica mínimamente invasiva de fijación trans-sacroilíaca.

Palabras clave: Dolor lumbar bajo, disfunción sacroilíaca, dolor articulación sacroilíaca, denervación sacroilíaca, inyección intraarticular, inyección articulación sacroilíaca.

\section{Anatomía}

La ASI es la articulación axial más grande del cuerpo, con área de $17,5 \mathrm{~cm}^{2}$, presenta gran variabilidad entre individuos en forma y tamaño. Corresponde a una articulación vertical, entre el sacro lateral y el ilioposteromedial, conectando el esqueleto axial con el apendicular. La articulación diartrodial verdadera comprende el $1 / 3$ anterior de la interfase entre sacro e ilion, correspondiendo el resto a una sindesmosis.

\section{Características articulares:}

La ASI tiene características únicas, atípicas respecto de otras articulaciones diartrodiales:

1. Fibrocartílago - superficial al cartílago hialino.

2. Superficies articulares variablemente onduladas, con muchas crestas y depresiones que minimizan el movimiento y aumentan la estabilidad, las superficies articulares se interbloquean entre sí1.

3. Cápsula articular fibrosa, rudimentaria y muy compacta, contiene escaso líquido articular. La historia natural de la cápsula articular es convertirse en una estructura de colágeno rígida e ir a la anquilosis fibrosa ${ }^{1}$.
4. Ligamentos adyacentes potentes y múltiples, brindan la estabilidad primaria por su tensión, limitando la movilidad. El complejo ligamentario posterior tiene forma de ventilador e incluye cuatro tipos de ligamentos diferentes que evitan la separación de la articulación y el movimiento de la pelvis a lo largo de varios ejes del sacro, manteniéndolo abrazado cuando el peso es transferido desde el torso a las extremidades inferiores ${ }^{1}$.

5. Estructuras miofasciales: Influyen en el movimiento y en la estabilidad, brindando soporte. Incluyen músculo dorsal ancho, vía fascia toracolumbar, glúteo mayor y piriforme.

\section{Inervación articular:}

Actualmente se dispone de escasa información, siendo mayor para la articulación posterior.

La articulación posterior estaría inervada principalmente por ramos dorsales laterales de S1 a S3, con aportes de L5 y S4. Destaca la alta variabilidad de la convergencia de los ramos laterales en la forámina, entre paciente $\mathrm{y}$ paciente, de lado a lado y entre un nivel y otro. No son visibles por imagenología ${ }^{2,3}$. 
La articulación anterior es inervada por ramos ventrales de L4-S2; Nakagawa (1966) afirma que desde L2 a S2, vía plexo lumbosacro del cual está muy próximo. Además, tiene contribuciones de nervios glúteo superior y obturador.

\section{Biomecánica}

La transición entre la columna y las extremidades inferiores está sometida a fuerzas de cizallamiento importantes. La función principal de la ASI es brindar estabilidad; mediante la transmisión y disipación de carga desde el tronco a las extremidades inferiores.

La forma de ventilador del complejo ligamentario posterior divide las fuerzas comunicadas por la columna al sacro transfiriéndolas a las dos hemipelvis y caderas. La ASI transfiere $80 \%$ de las fuerzas transversalmente al acetábulo y $20 \%$ verticalmente.

La ASI rota en los tres ejes durante el movimiento; las superficies articulares se deslizan una sobre la otra para absorber y resistir a las cargas mecánicas, presentando movilidad muy limitada. Los movimientos de rotación alcanzan entre 1 a $12^{\circ}$, y traslación, de 3 a $16 \mathrm{~mm}^{4}$. La amplitud del movimiento es $40 \%$ menos en hombres que en mujeres. Los hombres tienen más movimientos de traslación y las mujeres más movimientos de rotación. La influencia hormonal aumenta la laxitud ligamentaria, brindando hipermovilidad relativa de ASI para el parto.

Se describen dos movimientos de rotación sobre el eje transversal:

- Nutación: o rotación ventral-movimiento de la base sacra anteroinferior en relación al íleon, desplazamiento posterior del extremo distal del sacro- se produce con la extensión lumbosacra, la flexión de cadera facilita la nutación.

- Contranutación: o rotación dorsal-movimiento de la base sacra posterosuperior en relación al íleon, desplazamiento anterior del extremo distal del sacro- se produce con la flexión lumbosacra, se facilita con la extensión de la cadera.
No hay diferencias entre movimientos rotacionales y traslacionales en articulaciones sintomáticas y asintomáticas ${ }^{5}$.

\section{Etiología}

Los mecanismos de daño son por la combinación entre carga axial y rotación, siendo la ASI más vulnerable que la columna lumbar a ambas.

En el $40 \%$ a $50 \%$ de los casos se reconoce un factor traumático específico precipitante tal como levantar un objeto pesado, caída de nalgas, accidente de tránsito por detrás; o accidente de tránsito de tipo amplio, por colisión automovilística o caída inesperada en un hoyo.

La mitad de las causas son idiopáticas o por microtrauma, debidas a excesiva tensión de los ligamentos que aseguran la coaptación ASI, dolor por estrés mecánico de la cápsula y ligamentos de la ASI durante el embarazo, actividades deportivas o fusión lumbosacra.

Las causas de dolor SI se detallan en la Tabla 1.

\section{Entre los factores que predisponen a dolor SI están:}

a) Embarazo: en el cual se asocian el aumento de peso, postura lordótica exagerada y relajación ligamentosa durante el tercer trimestre más trauma pélvico asociado con el parto. La prevalencia de dolor SI en embarazadas en periodo de 9 meses es de $49 \%$. La inestabilidad puede persistir en mujeres multíparas de mediana edad, lo cual también es causa de dolor SI crónico ${ }^{6}$.

Tabla 1. Etiologías de dolor sacroilíaco

\begin{tabular}{|ll|}
\hline Intraarticular & Extraarticular \\
\hline Artritis: reumatoide y psoriática & Lesión ligamentosa \\
\hline Pelviespondilopatías & Fracturas óseas \\
\hline Cáncer & Cáncer \\
\hline Trauma & Dolor miofascial \\
\hline $\begin{array}{l}\text { Infección: estafilococia, } \\
\text { brucelosis, tuberculosis }\end{array}$ & Entesopatía \\
\hline Enfermedad quística & Trauma embarazo \\
\hline
\end{tabular}


b) Atletas y trabajadores manuales: Manteniendo postura asimétrica involuntariamente, debido a una técnica pobre cuando desarrollan su deporte como es el caso del patinaje o el jogging, o en trabajadores que realizan actividades repetitivas, que implican cizallamiento pélvico unidireccional y/o fuerzas torsionales ${ }^{7}$.

c) Dismetría: La presencia de discrepancia del largo extremidades de más de $5 \mathrm{~mm}$ provoca stress y vectores anormales de fuerza en la extremidad inferior ipsilateral ${ }^{8}$.

d) Anatomía transicional: La sacralización de L5 predispone a dolor SI ${ }^{9}$.

e) Escoliosis que se acompañan de desnivel pelviano ${ }^{10}$.

f) Anomalias de la marcha ${ }^{11}$.

g) Cirugía espinal: Especialmente cirugías que incluyen la fusión lumbosacra. La degeneración de la ASI aumenta al doble en el grupo con fusión espinal versus sin fusión a los 5 años, siendo mayor la incidencia en fusiones que se extienden hasta el sacro debido a que la fusión lumbosacra elimina todas las capacidades de adaptación en la unión L5-S1, transfiriendo el stress a ASI. El incremento de la degeneración de ASI postfusión se asocia a aumento del dolor SI entre 32\% y $61 \%{ }^{12}$.

\section{Epidemiología}

La articulación sacroilíaca representa una causa importante de dolor lumbar mecánico en pacientes de todas las edades, con distribución bimodal siendo más frecuente en viejos y jóvenes atletas. Se estima su frecuencia entre el $15 \%$ y $30 \%$ de los pacientes con dolor lumbar ${ }^{13}$.

Dentro de los pacientes que experimentan síntomas de dolor SI, tras confirmación con tres pruebas de provocación presentes y un bloqueo diagnóstico, solo el 16\% con síntomas de dolor SI tienen dolor efectivamente de origen $\mathrm{SI}^{14}$.

\section{Diagnóstico clínico}

\section{Historia}

Las características del dolor SI varían en forma importante de paciente en paciente, haciendo el diagnóstico difícil.
El dolor se localiza más frecuentemente en la región glútea. Fortín hizo un mapa del dolor lateral y caudal a espina iliaca posterosuperior $^{15}$. Slipman estudió 50 pacientes de dolor SI confirmado y observó que presentaban dolor glúteo en $94 \%$ de los casos, dolor lumbar en $72 \%$, dolor de extremidades inferiores en $50 \%$, especialmente muslo posterior y lateral, $28 \%$ de los pacientes reportó dolor bajo la rodilla, incluidos $14 \%$ que manifestaron dolor en el pie, $14 \%$ de los pacientes refirieron dolor inguinal ${ }^{16}$.

Young determinó tres criterios para sospechar que el dolor es de origen SI; dolor unilateral, que aumenta al pararse desde la posición sentada y ausencia de dolor lumbar ${ }^{17}$ $\mathrm{y}$ tres pruebas sacro ilíacas positivas.

El dolor tipo radicular que se observa en algunos pacientes con dolor SI se puede explicar por la cercanía de ASI a estructuras neurales, como raíces sacras y plexo lumbosacro.

El grado de repercusión funcional secundario al dolor sacroilíaco se realiza aplicando la Escala de Incapacidad por Dolor Lumbar de Oswestry (ODI), publicada en 1980 y validada al español ${ }^{18}$. Su utilización permite al clínico obtener información desde la perspectiva del paciente, conocer el grado de eficacia de las distintas técnicas de tratamiento empleadas y comparar resultados con otros estudios publicados.

\section{Examen físico}

Se debe evaluar el patrón de marcha, la diferencia del largo de extremidades y realizar examen de columna lumbar baja. El dolor SI raramente se observa en la línea media o sobre la espinosa de L5. Evaluar la movilidad de cadera y presencia de signo de Trendelemburg y realizar examen neurológico para descartar patología radicular lumbar.

Las pruebas SI se muestran en la Tabla 2.

Se ha observado una amplia variabilidad de la sensibilidad y especificidad de cada prueba, no siendo útiles en forma aislada. La presencia de 3 o más maniobras provocativas positivas tienen una sensibilidad de $94 \%$ y especificidad de $78 \%$, tras un bloqueo diagnóstico ${ }^{19}$, y sensibilidad de $85 \%$, especificidad $79 \%$, VPP 77\% y VPN $87 \%$ tras doble bloqueo 
Tabla 2. Pruebas sacroilíacas

\begin{tabular}{|l|}
\hline Prueba del dedo de Fortín \\
\hline Prueba de flexión o prueba de Gillet (Flexion Standing test) \\
Prueba de Patrick (FABER) \\
Prueba de Gaenslen \\
Prueba de distracción \\
Prueba de compresión \\
Prueba de empuje de muslo o prueba de Ostgaard \\
Prueba de empuje sacro \\
Prueba de Yeoman \\
Prueba de abducción resistida (REAB) \\
Prueba de elevación activa de la pierna extendida (Lasegue \\
activo) \\
\hline
\end{tabular}

diagnóstico, consistente en realizar dos bloqueos con anestésico local separados por una semana con alivio de los síntomas y signos sacro ilíacos, con alto poder discriminatorio (OR 17,16). De esta forma, la presencia de 3 o más maniobras provocativas positivas parecen tener una sensibilidad y especificidad razonable, identificando a aquellos individuos que responderán positivamente a inyecciones diagnósticas $\mathrm{SI}^{20}$.

\section{Exámenes complementarios}

Todos los pacientes con sospecha de dolor sacroiliaco deben tener:

1) Exámenes de laboratorio para descartar etiología inflamatoria, infecciosa y metabólica.

2) Estudios neurofisiológicos: Ante la sospecha de radiculopatía lumbar o neuralgia del pudendo, es recomendable realizar estudios de electromiografía, neuroconducción y potenciales evocados.

3) Estudios imagenológicos: La elección del estudio radiológico dependerá de la presentación clínica y la historia de cada paciente.

- Radiografía de Pelvis AP: Tiene muy baja sensibilidad y alta especificidad.

- TAC de pelvis: Considerado el gold standard para patología ósea, con sensibilidad $57,5 \%$ y especificidad $69 \%$, su realización está indicada en pacientes con dolor subagudo o crónico, lesiones mecánicas e inflamatorias ${ }^{21}$.

- RMI pelvis ósea: Efectivo para detectar etapas precoces de espondiloartropatías con sensibilidad mayor a $90 \%$, patología extraarticular de partes blandas, pero no es útil en condiciones no inflamatorias. Se usa en dolor de reciente comienzo y en pacientes jóvenes.

- Cintigrama óseo: Presenta alta especificidad $(100 \%)$, pero muy baja sensibilidad $(13 \%)$.

- Ecografía: Se utiliza en la detección de patología ligamentosa posterior, útil en embarazadas. La imagen Doppler de la red vascular en la articulación puede proveer diagnóstico de sacroileitis activa.

\section{Bloqueos para confirmación diagnóstica}

Tiene como objetivos la reproducción de los síntomas por distensión de la cápsula articular y la mitigación de los síntomas por bloqueo con anestésico local, logrando alivio dramático del dolor.

Son candidatos aquellos pacientes que presenten historia clínica compatible y tres o más maniobras provocativas positivas.

Dentro de las limitaciones de este método se señala que una inyección intraarticular verdadera es a veces difícil de obtener y las inyecciones intraarticulares diagnósticas pueden obviar estructuras periarticulares dolorosas ${ }^{22}$.

Dado que un bloqueo articular diagnóstico lleva a falsos positivos en el $20 \%$ de los casos, la IASP recomienda realizar dos bloqueos diagnósticos. La fluoroscopía y la ecografía son equivalentes, depende del entrenamiento $\mathrm{y}$ equipos con que se cuente.

Para considerar la prueba positiva la tasa de alivio debe alcanzar 50\% a 75\% ${ }^{23,24}$.

\section{Diagnóstico diferencial}

El diagnóstico diferencial con otras patologías de la región pelviana es, con frecuencia, difícil.

La patología de cadera es a menudo difícil de diferenciar de dolor SI ya que muchas maniobras SI también movilizan la cadera. El dolor de los órganos intrapélvicos también puede ser referido a la ASI. El síndrome del 
Tabla 3. Diagnóstico diferencial de dolor sacroilíaco

1) Dolor torácico y lumbar bajo
2) Patología discal lumbar
3) Dolor secundario a patología de cadera
4) Dolor originado en órganos pélvicos: gastrointestinal,
genitourinario, ginecológico
5) Dolor ligamento iliolumbar
6) Síndrome dolor miofascia: glúteo, cuadrado lumbar,
iliopsoas, multifidos, recto abdominal y piriforme
7) Síndrome piriforme
8) Neurológica:
- Radiculopatía lumbar
- Neuralgia del pudendo
9) Ostrapamiento nervios clúneos superiores
10) Tumor

piriforme puede coexistir con trastorno de la ASI o existir en forma aislada. La Tabla 3 muestra las patologías que deberían considerarse en el diagnóstico diferencial de dolor SI.

\section{Tratamiento}

\section{Manejo conservador}

En dolor SI se distinguen tres etapas:

- Etapa aguda: 1 a 3 días. El manejo incluye reposo relativo, aplicación de hielo/ movilización con calor, antiinflamatorios no esteroidales.

- Etapa de recuperación: de 3 días a 8 semanas. El tratamiento consiste en movilización articular y terapia física.

- Etapa de mantención: terapias dirigidas a corregir la dinámica postural. Una vez que el dolor ha cedido, intentar restaurar la mecánica normal.

\section{Kinesiterapia}

Los objetivos de la terapia física son: dores.

- Aliviar el dolor, eliminando sus genera-

- Aumentar la coaptación y estabilidad ASI, fortaleciendo la musculatura del cuádriceps, abdominales, isquiotibiales ${ }^{25-27}$.
- Mejorar déficits de flexibilidad, propiocepción, fuerza y problemas biomecánicos; desbalances maladaptativos, corrigiendo los mecanismos lumbopélvicos y de cadera ${ }^{25-27}$.

- Mejorar control postural dinámico ${ }^{28,29}$.

El programa de ejercicios diseñados para aliviar el dolor y corregir los problemas biomecánicos debería ser titulados individualmente.

El único punto validado en kinesiterapia es tener cuádriceps, abdominales e isquiotibiales bien desarrollados, necesarios para asegurar la coaptación y estabilidad de la articulación sacroilíaca, y que ellos controlan activamente.

\section{2. Órtesis}

a) Cinturones pélvicos u órtesis de estabilización pélvica. Dan confianza y conciencia propioceptiva en pacientes con disfunción sacroilíaca. Pueden limitar el movimiento y dar alivio en la etapa aguda, reduciendo las fuerzas de cizallamiento a través de la articulación y el stress alrededor de los músculos que la rodean, mejorando de este modo el dolor SI. Se recomienda destetar rápidamente del cinturón para evitar dependencia y debilidad, con posterior atrofia muscular ${ }^{30}$.

b) Taping (Water-resistant tape). Se consideran dispositivos de "palanca" antigravedad que supuestamente rotan el sacro en sentido anterior y el ilion en sentido posterior ${ }^{31,32}$.

El paso a una conducta intervencionista se determina por la No respuesta al tratamiento conservador o que han alcanzado un 'plateau' insatisfactorio tras 4 a 6 semanas de terapia, o antes en casos de dolor SI que limita el trabajo o en presencia de circunstancias extenuantes.

\section{Inyecciones intra o periarticulares}

La inyección intraarticular (IA) de la ASI puede afirmar el diagnóstico evitando cirugía innecesaria, reducir el dolor y facilitar la rehabilitación.

Se describen varias formas de realizar la infiltración:

- A ciegas o por referencia anatómica; es improbable alcanzar el espacio articular. Estudio con intervencionistas espinales "experimentados" a ciegas reveló que solo el 
$12 \%$ alcanzaba la articulación ${ }^{33}$. Otro estudio demostró que solo el $22 \%$ de los pacientes recibían una inyección IA cuando se realizaba solamente por palpación ${ }^{34}$.

- Bajo visión de fluoroscopía: Accediendo al aspecto inferior de la articulación por vía posterior que opone menos resistencia a la aguja; se realiza instilación de medio de contraste en el receso inferior, y posterior a ello, se infiltra con anestésico local.

- Bajo visión ecográfica: Diversos estudios han demostrado que es equivalente en agudeza a la fluoroscopía, pero tiene la ventaja de no someter al operador ni al paciente a radiación ionizante, ser más económica, más rápida y más accesible.

Dentro de la medicación a administrar se describen:

\section{Corticoesteroides (CE)}

CE mezclados con anestésicos locales han demostrado ser superiores a placebo, con alivio del dolor en $62 \%$ de pacientes a 3 meses y del $58 \%$ a los 6 meses $^{35}$. En dos estudios clínicos realizados en pacientes con dolor SI, el uso de CE ha mostrado ser superior al uso de anestésicos locales solos ${ }^{36}$. El número máximo recomendado es tres inyecciones de corticoides en 6 meses y cuatro en 1 año.

\section{Proloterapia}

Descrita como terapia proliferativa, consiste en inyectar agentes irritantes químicos en tejido ligamentario para producir inflamación, aumentar el flujo sanguíneo e incitar la proliferación de colágeno y cicatrización, con reparación tisular. Teóricamente la cicatrización y el engrosamiento de los ligamentos resulta en la estabilización de la articulación. El agente más utilizado es la dextrosa hiperosmolar al 25\%. En estudio realizado en 48 pacientes, comparando dextrosa bisemanal -4 dosis- versus CE IA, se observó alivio del dolor a los 15 meses de seguimiento en $58,7 \%$ con dextrosa versus $10,2 \%$ de los pacientes con $\mathrm{CE}^{37}$. En otro estudio, al realizar tres inyecciones de dextrosa hipertónica sobre ligamentos, se logró una tasa de éxito de $76 \%$ a los 12 meses y de $32 \%$ a los 24 meses $^{38}$. Los usos más recomendados para esta terapia peri o intraarticular es la disfunción sacroilíaca, por laxitud ligamentaria sacra. No hay estudios de proloterapia versus placebo, motivo por el cual se recomienda precaución en la interpretación de los resultados.

\section{Plasma rico en plaquetas (PRP)}

PRP autólogo inyectado para modular la vía antiinflamatoria y reparar el proceso del tejido dañado por macrófagos activados e inducir la proliferación de colágeno, diferenciación celular, vasodilatación y vascularización en áreas pobremente vascularizadas. En un estudio comparativo de PRP con CE en 40 pacientes se obtuvo alivio del dolor superior a los 3 meses con mejoría funcional en grupo de $\mathrm{PRP}^{39}$.

\section{Toxina botulínica (TB)}

En estudio realizado en 39 pacientes con toxina botulínica 100 UI periarticular (Dysport $^{\mathbb{R}}$ ) versus $\mathrm{CE}$ (triamcinolona) y anestésicos locales en ligamentos posteriores se observó mayor efecto en el grupo TB a los 2 y 3 meses de seguimiento, con reducción de $69 \%$ de la intensidad del dolor ${ }^{40}$.

\section{Precauciones}

Se ha observado que la eficacia de las infiltraciones es baja en pacientes con historia de dolor lumbar o fusión lumbosacra.

Dentro de las complicaciones reportadas después de la infiltración de ASI destacan la reacción vasovagal, prurito en sitio de punción, exacerbación del dolor, flush facial y/o sudoración varios días después, herpes simple y gangrena caseosa $\mathrm{a}^{41-43}$.

\section{Neurotomía por radiofrecuencia (RF)}

El alivio significativo pero temporal siguiendo las infiltraciones intraarticulares con CE pueden apoyar la indicación de neurotomía por radiofrecuencia.

La denervación RF de ramos sacros dorsales laterales, es más efectiva para aliviar dolor ASI extraarticular.

El criterio de éxito de la denervación por RF es $\geq 50 \%$ de alivio del dolor por un periodo $\geq 6$ meses. 
Tabla 4. Razones de falla de tratamiento intervencionista en dolor SI

\begin{tabular}{|c|c|c|}
\hline Selección del paciente & Diagnóstico inadecuado & Fallas técnicas de tratamiento \\
\hline $\begin{array}{l}\text { - Alta intensidad de dolor } \\
\text { preprocedimiento }\end{array}$ & $\begin{array}{l}\text { - Bloqueo falso positivo (20\% con un } \\
\text { solo bloqueo)* }\end{array}$ & - Aplicación de lesión pobre \\
\hline - Adulto mayor & $\begin{array}{l}\text { - Dolor articular ASI intraarticular y } \\
\text { ventral }\end{array}$ & - Complicaciones de procedimiento \\
\hline - Terapia opioide en altas dosis & - Generadores de dolor coexistentes & \\
\hline - Ganancia secundaria & & \\
\hline - Factores sociales & & \\
\hline - Patología psiquiátrica preexistente & & \\
\hline
\end{tabular}

* Hacer doble bloqueo previo a RF.

Las razones de falla del tratamiento intervencionista por radiofrecuencia se detallan en la Tabla 4.

Las limitaciones de la RF son la falta de efecto en las fibras nerviosas sensitivas de la parte anterior de ASI, producir área de lesión pequeña $(4 \mathrm{~mm})$, en una anatomía variable con nervios no visibles por imagenología y la tendencia a regeneración de los nervios tras varios meses post RF.

\section{Tipos de denervación}

1) RF Convencional: En estudio realizado en 43 pacientes, a los 3 meses se observó $67,5 \%$ de alivio del dolor $\geq 50 \%{ }^{44}$.

2) RF Bipolar: Uso de un segundo electrodo a $1 \mathrm{~cm}$ del primero para que fluya la corriente entre ambos y crear una lesión continua.

3) Sonda de RF simple multielectrodo: Posicionado por una entrada única a lo largo del sacro, lateral a la foramina sacra y medial a la ASI. En estudio en 102 pacientes se logró alivio del dolor $\geq 50 \%$ a los 3 meses de $81,6 \%$, 6 meses de $59,2 \%$ y al año de $35,7 \%{ }^{45}$.

4) $R F$ enfriada: Electrodos grandes internamente fríos que, al ser irrigados, permiten subir lentamente la temperatura, evitando quemar el tejido, obteniendo dos veces más extensión de la lesión. $57 \%$ de los pacientes sometidos a RF enfriada L4 a S3 logran analgesia $\geq 50 \%$ a los 6 meses, con duración de efecto de 8 meses ${ }^{46}$. Al comparar RF enfriada versus placebo se observó $59 \%$ de alivio $\geq 50 \%$ a los 9 meses, 3 veces más que placebo. Entre las desventajas RF enfriada se encuentran su alto costo, mayor tiempo de lesión, usar electrodos más gruesos con mayor riesgo de sangrado y dolor relacionado con el procedimiento y mayor incidencia de parestesias cutáneas. No hay ventajas significativas entre RF convencional y enfriada ${ }^{47}$.

5) $R F$ Pulsada (RFP): En estudio realizado en 22 pacientes se obtuvo $73 \%$ alivio del dolor $\geq 50 \%$ con duración de 5 meses $^{48}$.

Dentro de las complicaciones de la RF se describen la disestesia transitoria e hipoestesia sobre glúteo en el $20 \%$ de los pacientes, presencia de neuritis, sangrado e infección y desplazamiento de electrodos con incontinencia y empeoramiento del dolor o debilidad de las extremidades inferiores.

En metaanálisis de efectividad de RF, tras revisar 10 artículos, se concluyó que la RF es tratamiento efectivo del dolor SI a los 3 y 6 meses $^{49}$.

En estudio de 60 pacientes, al comparar dos inyecciones corticoides IA más anestésico local en 7 días versus RF bipolar de S1 a S3, a los 3 y 12 meses los grupos de RF reportaron más mejoría del dolor, mientras los con inyecciones IA no tuvieron una disminución significativa de éste ${ }^{50}$.

\section{Cirugía}

\section{Artrodesis o fusión SI}

Son candidatos a fusión SI los pacientes refractarios a tratamientos conservadores o terapias mínimamente invasivas como infiltraciones o RF. 
La cirugía parece claramente indicada postluxación o fractura, siendo su aplicabilidad controversial en enfermedades degenerativas, sin embargo, su utilización creciente en estas patologías hace deseable que el fisiatra esté informado de sus ventajas y complicaciones.

Existen dos tipos de técnicas, abierta y percutánea. La técnica percutánea consiste en la colocación de implantes de titanio bajo fluoroscopía, requiere menor tiempo quirúrgico, se asocia a disminución de la morbilidad, permite carga de peso desde el postoperatorio inmediato y determina breve periodo de rehabilitación; la tasa de fusión con esta técnica es de $89 \%$ a los 6 meses $^{51}$. En cambio, se opta por técnica abierta en caso de dismorfismo sacro, no unión o cirugía de revisión.

La tasa de complicaciones es similar en ambas técnicas, entre $18 \%$ y $21 \%$, son bursitis trocantereana, hematoma, infección, irritación radicular, pseudoartrosis, dolor relacionado con implante y embolia pulmonar. La tasa de reoperación después de cirugía abierta es de $15 \%$ y de $6 \%$ en percutánea ${ }^{52}$.

En revisión sistemática se resumen las distintas intervenciones en la articulación sacroilíaca $^{53}$.

\section{Conclusiones}

E1 dolor sacroilíaco es una patología compleja, de alta prevalencia, generalmente subdiagnosticada y subestimada en su magnitud, adscribiéndose generalmente dentro del dolor lumbar, lo cual frecuentemente la lleva a tratamientos prolongados e inefectivos. $\mathrm{Su}$ diagnóstico es difícil, generalmente clínico, requiere de la realización de una serie de maniobras de provocación que en su conjunto pueden ayudar al diagnóstico; sin embargo, ninguna de ellas es específica, y pacientes con patología lumbar baja o de cadera pueden referir dolor con ellas. Por este motivo frecuentemente es necesario realizar infiltraciones anestésicas para confirmar el diagnóstico. El tratamiento es variado, complejo y controversial, dada su etiología multicausal y frecuentemente desconocida.

\section{Referencias bibliográficas}

1. Bowen V, Cassidy JD. Macroscopic and microscopic anatomy of the sacroiliac joint from embryonic life until the eight decade. Spine 1981; 6: 620-8.

2. Grob KR, Neuhuber WL, Kissling RO. Innervation of the sacroiliac joint of the human. Z Rheumatol 1995; 54: 117-22.

3. Bernard T, Cassidy J. The Sacroiliac Joint Syndrome: Pathophysiology, Diagnosis, and Management. The Adult Spine: Principles and Practice. Raven Press Ltd, New York, NY, USA, 1991. p 2107-30.

4. Walker JM. The sacroiliac joint: a critical review. Phys. Ther. 1992; 72 (12): 903-16.

5. Robert R, Salaud C, Hamel O, Hamel A, Philippeau JM. Anatomie des douleurs de l'articulation sacroiliaque. Rev Rheum 2009; 76: 727-33.

6. Ostgaard HC, Andersson GB, Karlsson K. Prevalence of back pain in pregnancy. Spine 1991; 16 (5): 549-52.

7. Fortin JD, Flaco F. Enigmatic causes of spine pain in athletes. Phys Med Rehabil State Art Rev 1977; 11: 445-64.

8. Friberg O. Clinical symptoms and biomechanics of lumbar spine and hip joint in leg length inequality. Spine 1983; 8 (6): 643-51.

9. Avrahami E, Cohn DF, Yaron M. Computerized tomography, clinical and X-ray correlations in the hemisacralized fifth lumbar vertebra.Clin Rheumatol. 1986; 5 (3): $332-7$.

10. Timgren J, Soinila S. Reversible pelvic asymmetry: an overlooked syndrome manifesting as scoliosis, apparent leg-length difference, and neurologic symptoms. J Manipulative Physiol Ther 2006; 29 (7): 561-5.

11. Cibulka MT, Morr B, Wedel J, Bohr Z, Jones G, Herman C, Strube MJ. Changes in pelvic tilt during three different reciprocal stance positions in patients with sacroiliac joint regional pain. Int J Sports Phys Ther 2019; 14 (6): 967-77.

12. Ha KY, Lee JS, Kim KW. Degeneration of sacroiliac joint after instrumented lumbar or lumbosacral fusion: a prospective cohort study over five-year follow-up. Spine 2008; 33 (11): 1192-8.

13. Simopoulos TT, Manchikanti L, Singh V, Gupta S, Hameed H, Diwan S, et al. A systematic evaluation of prevalence and diagnostic accuracy of sacroiliac joint interventions. Pain Physician 2012; 15 (3): 305-44.

14. Schwarzer AC, Aprill CN, Bogduk N. The sacroiliac joint in chronic low back pain. Spine 1995; 20 (1): 31-7.

15. Fortin JD, Dwyer AP, West S, Pier J. Sacroiliac joint: pain referral maps upon applying a new injection/ar- 
thrography technique. Part I: asymptomatic volunteers. Spine 1994; 19 (13): 1475-82.

16. Slipman CW, Lipetz JS, Plastaras CT, Jackson HB, Vresilovic EJ, Lenrow DA, et al. Fluoroscopically guided therapeutic sacroiliac joint injections for sacroiliac joint syndrome. Am J Phys Med Rehabil 2001; 80: 425-32.

17. Young S, Aprill C, Laslett M. Correlation of clinical examination characteristics with three sources of chronic low back pain. Spine J. 2003; 3 (6): 460-5.

18. Flórez García MT, García Pérez MA, García Pérez F, Armenteros Pedreros J, Álvarez Prado A, Martínez Lorente MD. Adaptación transcultural a la población española de la escala de incapacidad por dolor lumbar de Oswestry. Rehabilitación (Madr). 1995; 29: 138-45.

19. Laslett M, Aprill CN, McDonald B, Young SB. Diagnosis of sacroiliac joint pain: Validity of individual provocation tests and composites of tests. Man Ther. 2005; 10 (3): 207-18.

20. van der Wurff P, Buijs EJ, Groen GJ. A multitest regimen of pain provocation tests as an aid to reduce unnecessary minimally invasive sacroiliac joint procedures. Arch Phys Med Rehabil. 2006; 87 (1): 10-4.

21. Elgafy H, Semaan HB, Ebraheim NA, Coombs RJ. Computed tomography findings in patients with sacroiliac pain. Clin Orthop Relat Res. 2001; 382: 112-8.

22. Kennedy DJ, Engel A, Kreiner DS, Nampiaparampil D, Duszynski B, MacVicar J. Fluoroscopically Guided Diagnostic and Therapeutic Intra-Articular Sacroiliac Joint Injections: A Systematic Review. Pain Med. 2015; 16 (8): 1500-18.

23. Lorio MP, Polly DW, Ninkovic I, Ledonio CGT, Hallas $\mathrm{K}$, Andersson G: Utilization of minimally invasive surgical approach for sacroiliac joint fusion in surgeon population of ISASS and SMISS membership. Open Orthop J 2014; 8: 1-6.

24. Manchikanti L, Singh V, Pampati V, Damron KS, Barnhill RC, Beyer C, et al. Evaluation of the relative contributions of various structures in chronic low back pain. Pain Physician 2001; 4 (4): 308-16.

25. Miller JAA, Schultz AM, Anderson GISJ. Loading displacement behaviors of sacroiliac joints. J Orthop Res 1987; 5: 92-101.

26. Vleeming A, Van Wingerden JP, Snijders CJ, Stoeckart R, Stijnen T. Load application to the sacrotuberous ligament. Influences on sacroiliac joint mechanics. Clin Biomech 1989; 4: 204-9.

27. Vleeming A, Stoeckart TR, Snijders CJ. The sacrotuberous ligament: A conceptual approach to its dynamic role in stabilizing the sacroiliac joint. Clin Biomech 1989; 4: 201-3.
28. Fortin JD. Sports specific stabilization: Figure skating. In White HA (ed), Basic science in the diagnosis and treatment of degenerative diseases of the spine. Volume I. CV Mosby Year-Book, Chicago, 1995.

29. Saal JA. Rehabilitation of sports related lumbar spine injuries. In Bellfus LC (ed), Physical Medicine and Rehabilitation, State Art Rev, Volume 1 (4), Hanley and Belfus, Philadelphia, 1987, pp 613-8.

30. Vleeming A, Buyruk HM, Stoeckart R, Karamursel S, Snijders CJ. An integrated therapy for peripartum pelvic instability: a study of the biomechanical effects of pelvic belts. Am J Obstet Gynecol. 1992; 166 (4): 1243-7.

31. Hoyt WH, Bard DA, Shaffer F. Experience with an antigravity leverage device of chronic low back pain: A clinical study. J Am Osteopath Assoc. 1981; 80: 474-9.

32. Neamat Allah N, Sigward S, Mohamed G, Elhafez S, Emran I. Effect of repeated application of rigid tape on pain and mobility deficits associated with sacroiliac joint dysfunction. J Back Musculoskelet Rehabil. 2019; 32 (3): 487-96.

33. Kissling RO. Zur arthrographis des iliosacralgelenks. Zeitschrift fur Rheumatologie 1992; 51: 183-7.

34. Rosenberg JM, Quint TJ, de Rosayro AM. Computerized tomographic localization of clinically-guided sacroiliac joint injections. Clin J Pain 2000; 16: 18-21.

35. Maugars Y, Mathis C, Berthelot JM, Charlier C, Prost A. Assessment of the efficacy of sacroiliac corticosteroid injections in spondylarthropathies: A double blind study. Br J Rheumato 1996; 35: 767-70.

36. Luukkainen RK, Wennerstrand PV, Kautiainen HH, Sanila MT, Asikainen EL. Efficacy of periarticular corticosteroid treatment of the sacroiliac joint in nonspondylarthropathic patients with chronic low back pain in the region of the sacroiliac joint. Clin Exp Rheumatol. $2002 ; 20$ (1): 52-4.

37. Kim WM, Lee HG, Jeong CW, Kim CM, Yoon MH. A randomized controlled trial of intra-articular prolotherapy versus steroid injection for sacroiliac joint pain. J. Altern Complement Med. 2010; 16 (12): 1285-90.

38. Cusi M, Saunders J, Hungerford B, Wisbey-Roth T, Lucas $\mathrm{P}$, Wilson $\mathrm{S}$. The use of prolotherapy in the sacroiliac joint. Br. J. Sports Med. 2010; 44 (2): 100-4.

39. Singla V, Batra YK, Bharti N, Goni VG, Marwaha N. Steroid vs. Platelet-Rich Plasma in Ultrasound-Guided Sacroiliac Joint Injection for Chronic Low Back Pain. Pain Pract. 2017; 17 (6): 782-91.

40. Lee JH, Lee SH, Song SH. Clinical effectiveness of botulinum toxin A compared to a mixture of steroid and local anesthetics as a treatment for sacroiliac joint pain. Pain Med. 2010; 11 (5): 692-700. 
41. Plastaras CT, Joshi AB, Garvan C, et al. Adverse events associated with fluoroscopically guided sacroiliac joint injections. PM R 2012; 4 (7): 473-8.

42. Meydani A, Schwartz RA, Foye PM, et al. Herpes simplex following intra-articular sacroiliac corticosteroid injection. Acta Dermatovenerol Alp Pannonica Adriat 2009; 18 (3): 135-7.

43. Kurnutala LN, Ghatol D, Upadhyay A. Clostridium sacroiliitis (gas gangrene) following sacroiliac joint injection-case report and review of the literature. Pain Physician 2015; 18 (4): E629-32.

44. Cheng J, Pope JE, Dalton JE, Cheng O, Bensitel A. Comparative outcomes of cooled versus traditional radiofrequency ablation of the lateral branches for sacroiliac joint pain. Clin J Pain. 2013; 29 (2): 132 7.

45. Buijs EJ, Kamphuis ET, Groen GJ. Radiofrequency treatment of sacroiliac joint related pain aimed at the first three sacral dorsal rami: a minimal approach. Pain Clin 2004; 16 (2): 139-46.

46. Bellini M, Barbieri M. Single strip lesions radiofrequency denervation for treatment of sacroiliac joint pain: two years' results. Anaesthesiol Intensive Ther 2016; 48 (1): 19-22.

47. Cohen SP, Hurley RW, Buckenmaier CC 3rd, Kurihara C, Morlando B, Dragovich A. Randomized placebo- controlled study evaluating lateral branch radiofrequency denervation for sacroiliac joint pain. Anesthesiology 2008; 109 (2): 279-88.

48. Vallejo R, Benyamin RM, Kramer J, Stanton G, Joseph NJ. Pulsed radiofrequency denervation for the treatment of sacroiliac joint syndrome. Pain Med 2006; 7: 429-34.

49. Aydin SM, Gharibo CG, Mehnert M, Stitik TP. The role of radiofrequency ablation for sacroiliac joint pain: a meta-analysis. PM R. 2010; 2 (9): 842-51.

50. Cánovas Martínez L, Orduña Valls J, Paramés Mosquera E, Lamelas Rodríguez L, Rojas Gil S, Domínguez García M. Sacroiliac joint pain: Prospective, randomized, experimental and comparative study of thermal radiofrequency with sacroiliac joint block. Rev Esp Anestesiol Reanim. 2016; 63 (5): 267-72.

51. Wise CL, Dall BE: Minimally invasive sacroiliac arthrodesis: Outcomes of a new technique. J Spinal Disord Tech 2008; 21: 579-84.

52. Zaidi HA, Montoure AJ, Dickman CA. Surgical and clinical efficacy of sacroiliac joint fusion: a systematic review of the literature. J Neurosurg Spine. 2015; 23 (1): 59-66.

53. McKenzie-Brown AM, Shah RV, Sehgal N, Everett CR. A systematic review of sacroiliac joint interventions. Pain Physician 2005; 8: 115-25. 